\title{
UKF-Based Underwater Terrain Matching Algorithms Combination
}

\author{
Feng Wei ${ }^{1, a}$, Zhangjing Yuan ${ }^{1, b}$, Rao Zhe ${ }^{1, c}$ \\ ${ }^{1}$ Department of Weaponry Engineering, Naval University of Engineering, Wuhan, 430033, China \\ aemail: 476507122@qq.com, bemail:wlamazing@163.com, 'email:icesnowaloe@163.com
}

Keywords: UKF; Underwater Terrain Matching Algorithm; Algorithm Combination; Underwater Terrain Based Navigation

\begin{abstract}
In order to neutralize the disadvantages of UKF algorithm applied in discontinuous terrain based navigation, this study offers an updated TERCOM/ICCP+UKF terrain matching algorithms combination. First, the theoretical and practical limitations of UKF-based underwater terrain matching algorithm adopted in terrain based navigation system are analyzed. Then, regarding the discounted effectiveness of algorithm matching resulted from larger initial navigation deviation, on the basis of commonly used "rough searching" and "precise matching" algorithm ideology, a combination approach of TERCOM, ICCP and UKF matching algorithms as well as the possible solution to similarly problematic terrains is illustrated. Simulation results show that algorithm combination has advantage over individual UKF matching algorithm because of offsetting the influence of larger initial navigation deviation on algorithm matching effectiveness, and presents better adaptability and applicability.
\end{abstract}

\section{Introduction}

The Unscented Kalman Filter (UKF) algorithm [1] is newly invented nonlinear filtering terrain matching algorithm. Since it is able to conduct real-time amendment to navigation system error and provide high matching precision under little initial position error, it is promising in engineering application. However, when conducting actual terrain based navigation, limited by prior underwater terrain data and detection equipment function, seldomly could the navigation cover the entire voyage of AUV. When AUV enters matching area, if the initial position provided is deviated, the convergence time needed for UKF will increase, so will the matching precision be influenced. Improvement just on UKF algorithm is not enough for enhance matching efficiency and somehow affects applicability. Therefore, if we can combine TERCOM/ICCP with UKF and use batch matching algorithm to offset the influence of huge initial position error, we may be able to improve applicability of UKF matching algorithm.

\section{Deficiencies of UKF underwater terrain matching algorithm}

In reality, short measurement range of sounding device, low resolution of digital map and the "matching blind zone" resulted from flat terrain will significantly undermine the performance of underwater terrain matching algorithm [2]. Oftentimes, we use route planning and intermittent matching to deal with such "blind zones". The latter has less requirement on AUV path and therefore more suitable for engineering application. Graph 1 is a diagrammatic sketch for intermittent matching.

Zone I and II are two matchable areas along designated path and the rest is "matching blind zone". Terrain matching navigation is adopted by AUV along route AB and then change to inertial navigation or other methods when it enters route BC. When it comes to site $\mathrm{C}$, terrain based navigation is back on. At this time, by referring to the provided position and navigation deviation, we are able to gradually amend the deviation by means of terrain matching algorithm. From the graph we can see that, when our AUV was launched at site A, the navigation deviation is small. So the terrain matching algorithm only need to scan a relatively small area to fulfill convergence; however, at site C, after a period of "blind" navigation, negative factors such as gyroscopic drift 
may accumulate larger position error for our AUV. Therefore, much larger area needs to be scanned for terrain matching algorithm to converge. Since the "blind zones" extensively exist and are unavoidable, matching performances with huge deviation like that at $\mathrm{C}$ site will appear multi-time along one designated path. Moreover, the convergence of UKF-based underwater terrain matching algorithm has to go through a certain time recurrence filtering process. With the enlarged initial navigation error, this process will also be prolonged. Given this thought, huge initial navigation deviation affects the efficiency of UKF algorithm directly, brings trouble to engineering application.

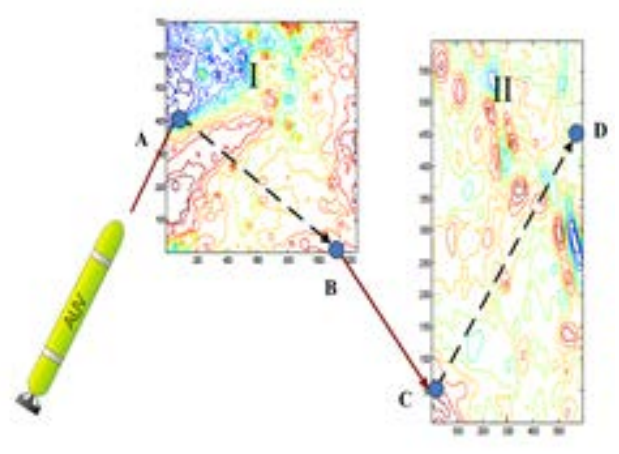

Graph 1 Intermittent terrain-based navigation diagram

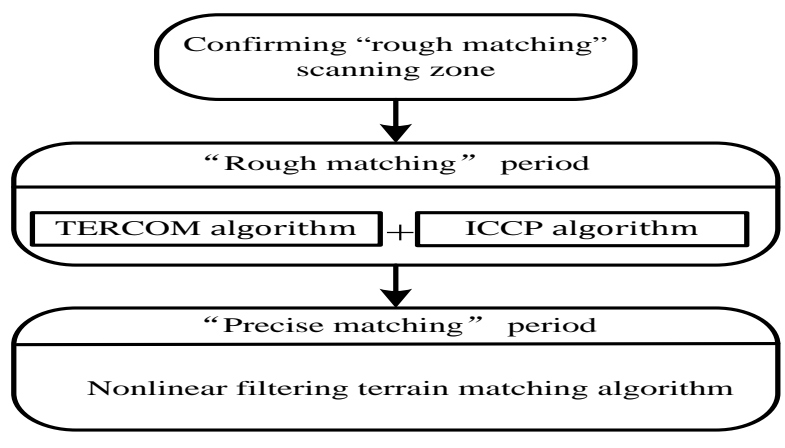

Graph 2 TERCOM $\backslash \mathrm{CCP}+\mathrm{UKF}$ combinative terrain matching algorithm procedure

\section{Analyses on combination modes of terrain matching algorithms}

Since improvements just on individual nonlinear filtering algorithms yield few outcomes in overcoming influences of big initial error, given different characteristics of each terrain matching algorithms, combinative application is more practical.

In this mode, the combinative algorithm needs to scan a large area during the "rough scanning" phrase. This phrase only aims at quickly narrowing down position deviation, so it doesn't require high resolution of the map. Based on the estimated result generated from "rough scanning" phrase, switches among different algorithms can help achieving matching algorithm with higher precision on high-resolution digital map.

This paper is going to combine TERCOM, ICCP together with UKF, with the view to come up with a more applicable combinative matching algorithm under big initial navigation error. To be more specific, TERCOM and ICCP are employed to correct the position error and course deviation from initial matching point, and ensure quick convergence for UKF terrain matching algorithm. That is TERCOM and ICCP are candidate algorithms for "rough scanning" phrase while UKF will be applied in "precise matching" period. The process goes like this: The "rough scanning" area is chosen by $3 \sigma$ principle. That is to open a $6 \sigma \times 6 \sigma$ scanning window centered on assigned navigation site. Before shift to "precise matching", judgment on the convergence of "rough scanning" should be made. That is to make sure the position error at that time is no larger than that at the beginning.

\section{TERCOM/ICCP+UKF mode}

In order to neutralize the negative influence of huge position error generated by TERCOM, based on relevant literature [3], this paper suggests to evaluate the precision of matching results through positions' variance distribution. First, set $K_{M S D}$ and $K_{C O N}$ as two threshold values with KMSD responsible for the number of similar topographic profile within scanning area, defined as follow:

$$
K_{\text {MSD }}=(1+v) \times \min \left\{M S D_{i}\right\}_{i=1}^{n}
$$

In this equation, $v$ is in control of the number of kept topographic profiles. The larger its value 
is, the more profiles are kept for subsequent calculation. If the mean variance $M S D_{i}<K_{M S D}$, we can say that this topographic profile resembles actual existence. If the coordinate of the first depth is represented as $\left(x_{i}^{1}, y_{i}^{1}\right)$, then the mean variance $\sigma_{K}$ of all the first depths of kept profiles can be calculated as:

$$
\sigma_{K}=\frac{\sum_{i=1}^{n} \sqrt{\left(x_{i}-\bar{x}\right)^{2}+\left(y_{i}-\bar{y}\right)^{2}}}{n}
$$

The value of $\sigma_{K}$ reflects the degree of dispersion of similar topographic profiles within scanning zone. If $\sigma_{K} \geq K_{\text {con }}$, their locations are dispersive, which means there is great possibility of the existence of similar terrains, like indicated in graph 4. So the precision of TERCOM matching outcomes can't be ensured. If $\sigma_{K}<K_{\text {con }}$, this area shows little variation, therefore the estimation error of TERCOM algorithm is smaller, as indicated in 4(b).

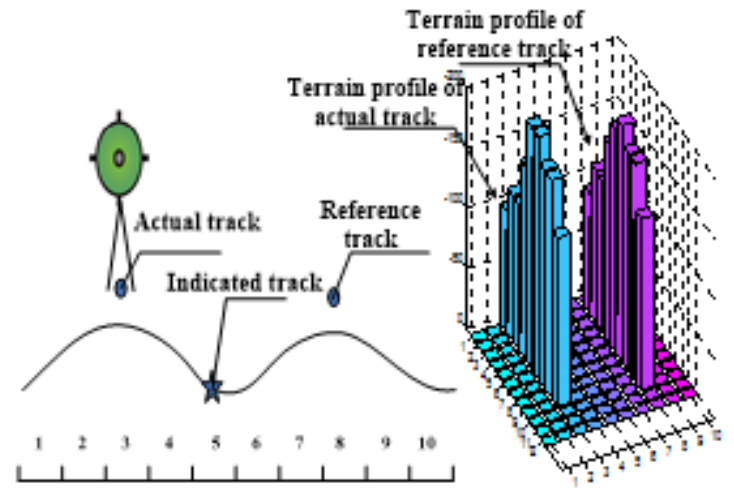

Graph 3 influences of TERCOM algorithm on similar terrain

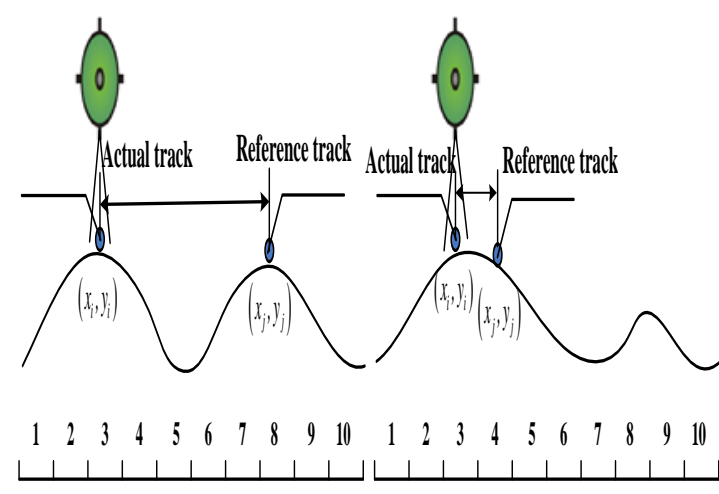

$\begin{array}{ll}\text { (a) } \sigma_{K} \geq K_{\text {con }} & \text { (b) } \sigma_{K}<K_{\text {con }}\end{array}$

Graph 4 Location estimation strategy

If $\sigma_{K}<K_{\text {con }}$, find the smallest $M S D$ profile among $n$ remaining topographic profile, and make its coordinate as the estimated outcome of this TERCOM algorithm. Detailed procedure of TERCOM $\backslash$ ICCP+UKF matching algorithm is as follow:
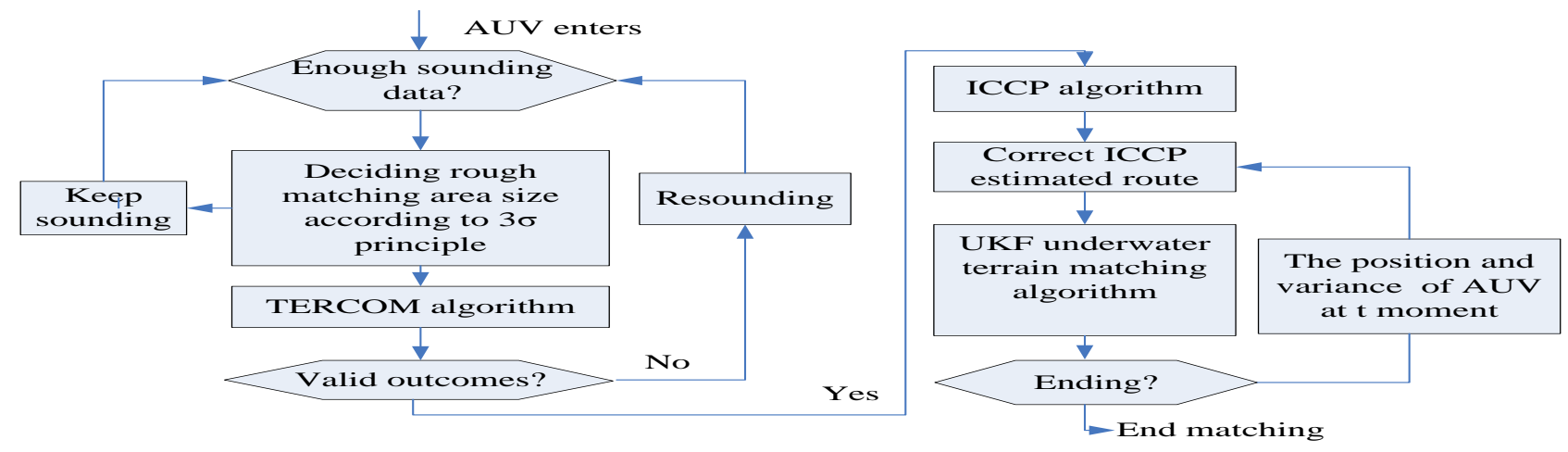

Graph 5 TERCOM $\backslash \mathrm{ICCP}+\mathrm{UKF}$ combinative algorithm flow diagram

\section{Simulated analysis}

Build underwater terrain matching model with the method presented by literature [4] with simulation parameters provided in figure 1 . Using digital map of $24 \mathrm{~m}$ resolution during "rough scanning" period helps accelerate calculation, while using that of $6 \mathrm{~m}$ resolution in "precise matching" helps increase matching precision. TERCOM's threshold value $v=0.1, K_{\text {con }}=40 \mathrm{~m}$, UKF's scaling factor $\lambda=1$.

The simulated results are provided in graph 6 , the vertical coordinates are the logarithmic 
coordinate system of $\log _{10}$.

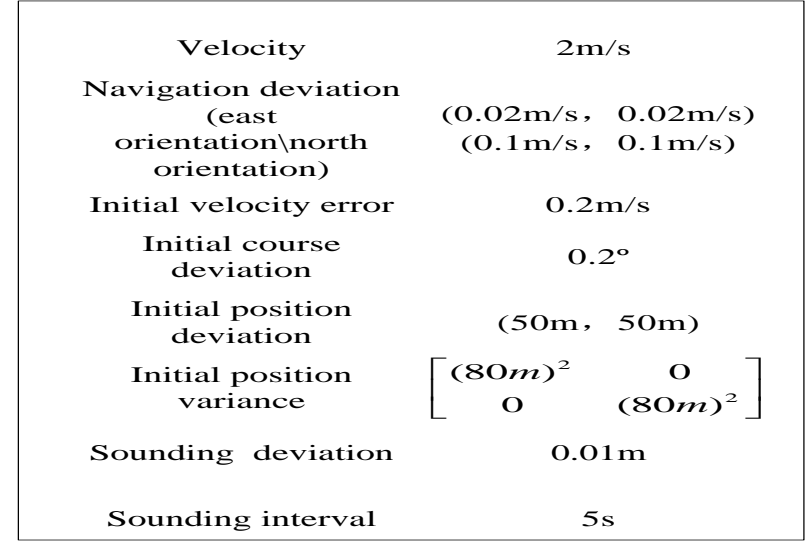

Figure 1 Simulation parameters

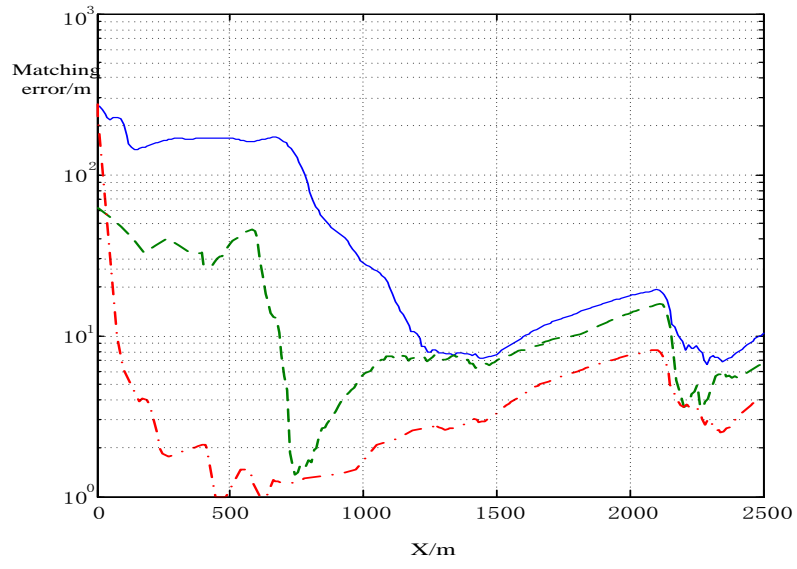

Graph 6 Combinative algorithm simulation outcome

In the diagram, Posterior Cramer-Rao Bound (PCRB) [5] is often used to evaluate the lower limit of terrain matching algorithm's optimal precision. The closer the matching algorithm resembles PCRB curve, the better the matching outcomes. From the diagram we can see that the error curve of combinative algorithm is closer to PCRB curve, which certifies to the effectiveness of the combined algorithm compared to individual UKF terrain matching algorithm. Since TERCOM and ICCP adopt batch approach, which is free from gradual convergence, the matching error of combined algorithm suddenly decreases in rough matching phrase, setting basis for subsequent quick convergence of UKF. Thus the negative affects of huge initial error on algorithm matching outcomes are well handled, which proves to the better adaptability of combined algorithm.

\section{Conclusion}

This paper introduces the basic principle of UKF-based underwater terrain matching algorithm and analyzes the practical limitations of underwater terrain navigation, pointing out the negative influences of huge navigation error on UKF's application. Given that the outcomes of improvements on individual UKF are unsatisfactory, this paper suggests an algorithm combination. Based on the commonly applied "rough scanning" and "precise matching” algorithm combination, a refined UKF-based TERCOM/ICCP+UKF algorithm combination is presented and verified in simulation.

\section{References}

[1] Julier S J. Unscented Filtering and Nonlinear Estimation [J]. Proc IEEE , 2004 , 92(3) : 401-422.

[2] Na Yuan. Study on Matching Algorithm of Terrain-Aided Navigation System [D]. Harbin: Harbin Engineering University, 2007.

[3] Kedong Wang, Yong Yang. Approaches and Equipments of Terrain-Aided Navigation [P]. China: CN101339036A, 2008-08-20.

[4]Shen Jian, Yan Ping, Jingyuan Zhang, A Method for Reconstructing 3D Seabed Digital Map Based Upon Image Processing[J], Computer Simulation , 2009, 26(10): 90-93.

[5]Bergman N.Recursive Bayesian Estimation Navigation and Tracking Applications [D]. Sweden: Linkoping University, 1999. 